\title{
A REMARK ON REFINABLE MAPS AND CALMNESS
}

\author{
HISAO KATO
}

\begin{abstract}
It is shown that if $r: X \rightarrow Y$ is a refinable map between compacta and $Y$ is calm, then $r$ is a shape equivalence. As a corollary, if $r: X \rightarrow Y$ is a refinable map between compacta and either $X$ or $Y$ is $S^{n}$-like $(n \geq 1)$, then $r$ is a shape equivalence, where $S^{n}$ denotes the $n$-sphere.
\end{abstract}

0. Introduction. In [9], J. Ford and J. W. Rogers, Jr. introduced the notion of refinable maps and they proved several results about these maps. In [10], we showed that every refinable map does not preserve shape (cf. [12]) and introduced the notion of pseudo-isomorphisms in pro-category, and by using this notion we proved that if $r: X \rightarrow Y$ is a refinable map between compacta and $Y$ is an FANR, then $r$ is a shape equivalence. In this paper, we will give the following more general result: if $r: X \rightarrow Y$ is a refinable map between compacta and $Y$ is calm, then $r$ is a shape equivalence. The notion of calmness was introduced by Z. Cerin [4]. It is well known that the dyadic solenoid is calm but not movable [4]. As a corollary, we obtain that if $r: X \rightarrow Y$ is a refinable map between compacta and either $X$ or $Y$ is $S^{n}$-like $(n \geq 1)$, then $r$ is a shape equivalence. In relation to the above result, the following are known; if $r: X \rightarrow Y$ is a refinable map between compacta, then $r$ is a weakly confluent map [9], moreover; $[14]$,

(1) if $Y$ has property $[K]$ due to J. L. Kelley $[15,(3.2)]$, then $r$ is a confluent map

(2) if $Y$ is locally connected, then $r$ is a monotone map [9],

(3) if $Y$ is locally $n$-connected $(n \geq 1)$, then $r$ is a $U V^{n}$-map [11], and

(4) if $Y$ is an ANR (locally contractible), then $r$ is a cell-like map [11].

Several properties concerning the notions of refinable maps, ARI-maps, AI-maps, calmness, AANR and quasi-ANR etc. have been studied in $[1,3-14,16,17$ etc.].

1. Definitions and notations. For a metric space $X$, if $x$ and $y$ are points of $X, d(x, y)$ denotes the distance from $x$ to $y$. A map $r: X \rightarrow Y$ between compacta is refinable [9] if for any $\epsilon>0$ there is a surjective map $f: X \rightarrow Y$ such that $\operatorname{diam} f^{-1}(y)<\epsilon$ for each $y \in Y$ and $d(r, f)=\sup \{d(r(x), f(x)) \mid x \in X\}<\epsilon$. Note that every refinable map is surjective, every near-homeomorphism is refinable and if there is a refinable map from a compactum $X$ to a compactum $Y$, then $X$ is $Y$-like. But simple examples show that the converse of any of these assertions is not true. A compactum $X$ is calm [4] if whenever $X \subset M \in$ ANR, there is a neighborhood $V$ of $X$ in $M$ such that for any neighborhood $U$ of $X$ in $M$ there is a neighborhood $W$ of $X$ in $M, W \subset U$, such that if $f, g: Y \rightarrow W$ are maps with $f \simeq g$ in $V$, then $f \simeq g$ in $U$ for all $Y \in \mathrm{ANR}$. Let $K$ be an arbitrary category. By $\mathbf{K}$, we mean the category of inverse systems in $K$ and system maps in $K$, also by pro- $K$, the

Received by the editors March 1, 1983 and, in revised form, June 3, 1983.

1980 Mathematics Subject Classification. Primary 54C10, 54C56, 55P55.

Key words and phrases. Refinable map, shape equivalence, calm, FANR, AANR, movable. 
homotopy category of $\mathbf{K}$. An inverse system $\left\{X_{\alpha}, p_{\alpha \alpha}, A\right\}$ of pro- $K$ is calm if there is $\alpha_{0} \in A$ such that

for any $\alpha \in A\left(\alpha \geq \alpha_{0}\right)$ there is $\beta \in A, \beta \geq \alpha$, such that

if $f, g: Y \rightarrow X_{\beta}$ are morphisms in $K$ with $p_{\alpha_{0} \beta} f=p_{\alpha_{0} \beta} g$, then $p_{\alpha \beta} f=p_{\alpha \beta} g$ for all $Y \in K$.

It is easily seen that a compactum $X$ is calm iff there is an inverse system $\left\{X_{n}\right.$, $\left.\left[p_{n n+1}\right]\right\}$ in pro-HCW which is calm and associated with $X$, where HCW is the category of spaces having the homotopy type of CW-complexes and homotopy classes of maps. A system map $\mathbf{f}=\left\{f, f_{\beta}, B\right\}:\left\{X_{\alpha}, p_{\alpha \alpha^{\prime}}, A\right\} \rightarrow\left\{Y_{\beta}, q_{\beta \beta^{\prime}}, B\right\}$ of $\mathbf{K}$ is a pseudo-isomorphism [10] if for each $\beta \in B$ and each $\alpha \geq f(\beta)$ there exist $g(\alpha, \beta) \geq \beta$ and a morphism $g_{(\alpha, \beta)}: Y_{g(\alpha, \beta)} \rightarrow X_{\alpha}$ such that

$(* *)$ for every $\beta^{\prime} \geq g(\alpha, \beta)$ there exist $h\left(\beta^{\prime}\right) \geq \alpha$ and a morphism $h_{\beta^{\prime}}: X_{h\left(\beta^{\prime}\right)} \rightarrow$ $Y_{\beta^{\prime}}$ such that

$$
f_{\beta} p_{f(\beta) \alpha} g_{(\alpha, \beta)}=g_{\beta g(\alpha, \beta)} \text { and } g_{(\alpha, \beta)} q_{g(\alpha, \beta) \beta^{\prime}} h_{\beta^{\prime}}=p_{\alpha h\left(\beta^{\prime}\right)} .
$$

A morphism $f: \mathbf{X}=\left\{X_{\alpha}, p_{\alpha \alpha^{\prime}}, A\right\} \rightarrow \mathbf{Y}=\left\{Y_{\beta}, q_{\beta \beta^{\prime}}, B\right\}$ of pro- $K$ is a pseudoisomorphism if it has a pseudo-isomorphism $\mathbf{f}: \mathbf{X} \rightarrow \mathbf{Y}$ of $\mathbf{K}$ as the representation, i.e. $f=[\mathbf{f}]$.

Theorem (1.1) [7]. A compactum $X$ is an FANR iff $X$ is calm and movable.

TheOREm (1.2) [1]. A compactum $X$ is an $A A N R_{N}$ iff $X$ is an $A A N R_{C}$ and an FANR.

2. Calmness and pseudo-isomorphisms. In this section, we show that if $r: X \rightarrow$ $Y$ is a refinable map between compacta and $Y$ is calm, then $r$ is a shape equivalence. First, we prove the following lemma (cf. [10, (2.1)].

Lemma (2.1). For a category $K$, if $f: \mathbf{X}=\left\{X_{\alpha}, p_{\alpha \alpha^{\prime}}, A\right\} \rightarrow \mathbf{Y}=\left\{Y_{\beta}, q_{\beta \beta^{\prime}}, B\right\}$ is a pseudo-isomorphism in pro- $K$ and $\mathbf{Y}$ is calm, then $f$ is an isomorphism in pro- $K$.

Proof. Let $\mathbf{f}=\left\{f, f_{\beta}, B\right\}: \mathbf{X} \rightarrow \mathbf{Y}$ be a pseudo-isomorphism of $\mathbf{K}$ such that $f=[\mathbf{f}]$. Since $\mathbf{Y}$ is calm, there is $\beta_{0} \in B$ satisfying the condition $(*)$. Let $\beta \geq \beta_{0}$. Since $\mathbf{f}$ is a pseudo-isomorphism, for each $\alpha \geq f(\beta)$ there exist $g(\alpha, \beta) \geq \beta$ and a morphism $g_{(\alpha, \beta)}: Y_{g(\alpha, \beta)} \rightarrow X_{\alpha}$ such that

$$
f_{\beta} p_{f(\beta) \alpha} g_{(\alpha, \beta)}=q_{\beta g(\alpha, \beta)}
$$

and the condition $(* *)$ is satisfied. By the choice of $\beta_{0}$, we can choose $\beta^{\prime} \geq$ $g(\alpha, \beta)$ such that if $f, g: Y \rightarrow Y_{\beta^{\prime}}$ are morphisms in $K$ with $q_{\beta_{0} \beta^{\prime}} f=q_{\beta_{0} \beta^{\prime}} g$, then $q_{g(\alpha, \beta) \beta^{\prime}} f=q_{g(\alpha, \beta) \beta^{\prime}} g$ for all $Y \in K$. Also, by the condition $(* *)$, there exist $h\left(\beta^{\prime}\right) \geq$ $f\left(\beta^{\prime}\right)$ and a morphism $h_{\beta^{\prime}}: X_{h\left(\beta^{\prime}\right)} \rightarrow Y_{\beta^{\prime}}$ such that

$$
g_{(\alpha, \beta)} q_{g(\alpha, \beta) \beta^{\prime}} h_{\beta^{\prime}}=p_{\alpha h\left(\beta^{\prime}\right)}
$$

and

$$
f_{\beta} p_{f(\beta) h\left(\beta^{\prime}\right)}=q_{\beta \beta^{\prime}} f_{\beta^{\prime}} p_{f\left(\beta^{\prime}\right) h\left(\beta^{\prime}\right)}
$$


Then by (1)-(3), we have

$$
\begin{aligned}
q_{\beta_{0}(\alpha, \beta)}\left(q_{g(\alpha, \beta) \beta^{\prime}} h_{\beta^{\prime}}\right) & =q_{\beta_{0} \beta} f_{\beta} p_{f(\beta) \alpha} g_{(\alpha, \beta)} q_{g(\alpha, \beta) \beta^{\prime}} h_{\beta^{\prime}} \\
& =g_{\beta_{0} \beta} f_{\beta} p_{f(\beta) \alpha} p_{\alpha h\left(\beta^{\prime}\right)} \\
& =q_{\beta_{0} \beta} f_{\beta} p_{f(\beta) h\left(\beta^{\prime}\right)} \\
& =q_{\beta_{0} \beta} q_{\beta \beta^{\prime}} f_{\beta^{\prime}} p_{f\left(\beta^{\prime}\right) h\left(\beta^{\prime}\right)} \\
& =q_{\beta_{0} g(\alpha, \beta)}\left(q_{g(\alpha, \beta) \beta^{\prime}} f_{\beta^{\prime}} p_{f\left(\beta^{\prime}\right) h\left(\beta^{\prime}\right)}\right) .
\end{aligned}
$$

By the condition $(*)$, we have

$$
q_{g(\alpha, \beta) \beta^{\prime}} h_{\beta^{\prime}}=q_{g(\alpha, \beta) \beta^{\prime}} f_{\beta^{\prime}} p_{f\left(\beta^{\prime}\right) h\left(\beta^{\prime}\right)} .
$$

Hence by (2) and (5), we have

$$
g_{(\alpha, \beta)} q_{g(\alpha, \beta) \beta^{\prime}} f_{\beta^{\prime}} p_{f\left(\beta^{\prime}\right) h\left(\beta^{\prime}\right)}=g_{(\alpha, \beta)} q_{g(\alpha, \beta) \beta^{\prime}} h_{\beta^{\prime}}=p_{\alpha h\left(\beta^{\prime}\right)} .
$$

By (1) and (6), we can conclude that the morphism $f: \mathbf{X} \rightarrow \mathbf{Y}$ is an isomorphism in pro- $K$.

Theorem (2.2) [10, 1.5 Theorem]. If $r: X \rightarrow Y$ is a refinable map between compacta, then $r$ induces a pseudo-isomorphism in pro- $H C W$.

By using (2.1) and (2.2), we obtain the following theorem (cf. [3, 3.3 Corollary]).

TheOREM (2.3). If $r: X \rightarrow Y$ is a refinable map between compacta and $Y$ is calm, then $r$ is a shape equivalence, i.e., $\operatorname{Sh}(X)=\operatorname{Sh}(Y)$.

Combining Theorems (1.1), (1.2) and (2.3), we have

Corollary (2.4) [10, 2.2 Theorem]. If $r: X \rightarrow Y$ is a refinable map between compacta and $Y$ is an FANR, then $r$ is a shape equivalence.

Corollary (2.5). If $r: X \rightarrow Y$ is a refinable map between compacta and $Y$ is an $A A N R_{N}$, then $r$ is a shape equivalence.

REMARK (2.6). In the statement of (2.3), we cannot replace "calm" by "movable", also in (2.5), we cannot replace $\mathrm{AANR}_{N}$ by $\mathrm{AANR}_{C}$ (see [10, Examples (2.5), (2.6) and (2.8)]).

Let $\mathfrak{P}$ be a class of ANR-sets. A compactum $X$ is $\mathfrak{P}$-calm [4] if whenever $X \subset$ $M \in \mathrm{ANR}$, there is a neighborhood $V$ of $X$ in $M$ such that for every neighborhood $U$ of $X$ in $M$ there is a neighborhood $W, W \subset U$, such that if $f, g: Y \rightarrow W$ with $f \simeq g$ in $V$, then $f \simeq g$ in $U$ for all $Y \in \mathfrak{P}$. By using this notion, we obtain a more general result than (2.3) as follows.

Theorem (2.7). If $r: X \rightarrow Y$ is a refinable map between compacta and $X$ is $\mathfrak{P}$-like and $Y$ is $\mathfrak{P}$-calm, then $r$ is a shape equivalence.

Proof. Observe the proofs of [10, 1.5 Theorem] and (2.3).

Corollary (2.8). If $r: X \rightarrow Y$ is a refinable map between compacta and if either $X$ or $Y$ is $S^{n}$-like $(n \geq 1)$, then $r$ is a shape equivalence, where $S^{n}$ denotes the $n$-sphere. 
Proof. By [9, Corollary 3.1], we conclude that $X$ and $Y$ are $S^{n}$-like. Let $\left\{Y_{i}, p_{i i+1}\right\}$ be an inverse sequence such that each $Y_{i}$ is the $n$-sphere $\left(=S^{n}\right)$ and $Y=$ inv $\lim \left\{Y_{i}, p_{i i+1}\right\}$. Note that $\pi_{n}\left(p_{i i+1}\right): \pi_{n}\left(Y_{i+1}\right) \rightarrow \pi_{n}\left(Y_{i}\right)$ is either a monomorphism or a zero homomorphism for each $i=1,2, \ldots$. Hence we can conclude that $Y$ is $S^{n}$-calm. Theorem (2.8) implies that $r$ is a shape equivalence.

We finish this paper with some open questions.

Question 1. Does every refinable map preserve calmness?

Question 2. Does every refinable map preserve FANR? (For the partial positive answer, see [10, Theorems 2.4, 2.12].)

Question 3. Does every refinable map preserve $\mathrm{AANR}_{N}$ ?

The positive answer of Question 1 implies the positive answer of Question 2 and the positive answer of Question 2 implies the positive answer of Question 3.

\section{REFERENCES}

1. S. A. Bogatyi, Approximate and fundamental retracts, Mat. Sb. 93 (1974), 91-103.

2. K. Borsuk, Theory of shape, PWN, Warsaw, 1975.

3. L. Boxer, AANR's and ARI maps, Topology Proc. 6 (1981), 219-226.

4. Z. Cerin, Homotopy properties of locally compact spaces at infinity-calmness and smoothness, Pacific J. Math. 79 (1978), 69-91.

5. __,$C_{p}$-e-movable and $C$-e-calm compact and their images, Compositio Math. 45 (1981), 115141.

6. Characterizing global properties in inverse limits, preprint.

7. Z. Cerin and A. P. Sostak, Some remarks on Borsuk's fundamental metric, Topology. I (Á. Czászár, ed.), North-Holland, New York, 1980, pp. 233-252.

8. M. Clapp, On a generalization of absolute neighborhood retracts, Fund. Math. 70 (1971), 117130.

9. J. Ford and J. W. Rogers, Jr., Refinable maps, Colloq. Math. 39 (1978), 263-269.

10. H. Kato, Refinable maps in the theory of shape, Fund. Math. 113 (1981), 119-129.

11. __ Refinable maps onto locally n-connected compacta, Tsukuba J. Math. 4 (1980), 83-87.

12. __ A note on refinable maps and quasi-homeomorphic compacta, Proc. Japan Acad. Ser. A Math. Sci. 58 (1982), 69-71.

13. __ A note on infinite-dimension under refinable maps, Proc. Amer. Math. Soc. 88 (1983), $177-180$.

14. _ Concerning a property of J. L. Kelley and refinable maps, preprint.

15. J. L. Kelley, Hyperspaces of a continuum, Trans. Amer. Math. Soc. 52 (1942), 22-36.

16. H. Noguchi, A generalization of absolute neighborhood retracts, Kodai Math. Sem. Rep. 1 (1953), 20-22.

17. P. Patten, Refinable maps and generalized absolute neighborhood retracts, Topology Appl. 14 (1982), 183-188.

Institute of Mathematics, University of TSUkUba, Ibaraki, 305 Japan 